\title{
Itinerary of High Density Lipoproteins in Endothelial
}

\section{Cells}

Damir Perisa ${ }^{1,2}$, Lucia Rohrer ${ }^{1,2}$, Andres Kaech ${ }^{3}$, Arnold von Eckardstein 1,2,*

1. Institute of Clinical Chemistry, University and University Hospital of Zurich, Switzerland

2. Competence Center for Integrated Human Physiology, University of Zurich, Switzerland

3. Center for Microscopy and Image Analysis, University of Zürich, Switzerland

${ }^{*}$ Corresponding author:

\author{
Arnold von Eckardstein \\ Institute of Clinical Chemistry \\ University Hospital Zurich \\ Rämistrasse 100, CH-8091 Zurich, \\ Switzerland \\ Tel.: $\quad 41-44-255-22-60$ \\ Fax.: $\quad 41-44-255-45-90$ \\ e-mail: arnold.voneckardstein@usz.ch
}

word counts: abstract: 234 words; main text: 3821 words; 9 figures, 44 references 


\section{Abstract (234 words)}

High density lipoprotein (HDL) and its main protein component apolipoprotein A-I (ApoA-I) have multiple anti-atherogenic functions. Some of them are exerted within the vessel wall, so that HDL needs to pass the endothelial barrier. To elucidate their itinerary through endothelial cells (ECs), we labelled ApoA-I and HDL either fluorescently or with $1.4 \mathrm{~nm}$ Nanogold and investigated their cellular localization by using immunofluorescent microscopy (IFM) and electron microscopy (EM). HDL as well as ApoA-I are taken up by ECs into the same route of intracellular trafficking. Time kinetics and pulse chase experiments revealed that HDL is trafficked through different vesicles. HDL partially co-localized with LDL, albumin, and transferrin. HDL did not colocalize with clathrin and caveolin-1. Fluorescent HDL was recovered at small proportions in early endosomes and endosome to trans-golgi network vesicles but not at all in recycling endosomes, in late endosomes or lysosomes. EM identified HDL mainly in large filled vesicles which however upon IFM did not colocalize with markers of multivesicular bodies or autophagosomes. The uptake or cellular distribution of HDL was altered upon pharmacological interference with cytochalasine D, colchicine and dynasore. Blockage of fluid phase uptake with Amiloride or EIPA did not reduce the uptake of HDL. Neither did we observe any co-localization of HDL with dextran as the marker of fluid phase uptake. In conclusion, HDL and ApoA-I are internalized and trafficked by endothelial cells through a non-classical endocytic route.. 


\section{Introduction}

The endothelium is a dynamic and selective barrier and its permeability is highly regulated under physiological and pathological conditions. In the subendothelial space of arteries, lipids and cholesterol as well as protein aggregates [1] can be stranded and accumulate extracellularly or in macrophages. Eventually, a plaque is formed in the intima leading to atherosclerosis. Many epidemiological studies showed an inverse correlation between high density lipoprotein (HDL) cholesterol concentration and cardiovascular events [2]. Therefore and due to its biological impacts HDL is believed to protect from atherosclerosis. Of note, $\mathrm{HDL}$ removes cholesterol from macrophage foam cells of the arterial wall for reverse transport to the liver [3,4]. Beyond this classical function, HDL has many anti-oxidative, antiinflammatory, and anti-thrombotic activities [5].

Many of these functions happen inside the vascular wall or other peripheral tissues rather than in the blood stream. Therefore, HDL has to pass the endothelium of blood $[6]$ and lymphatic vessels $[7,8]$ to enter and leave the extravascular compartments, respectively.

The endothelium is actively regulating the passage of any molecule or cell. Endothelial cells form the glycocalyx, which is a thick steric filter $[9,10]$ as well as an interaction platform for a variety of proteins in the blood stream. Furthermore, EC's regulate transcellular and paracellular transport by internalizing cargo through endocytic receptors, opening and closing of channels, as well as tight and adherence junctions.

Previously, we showed by biochemical methods and RNA interference that both lipid-rich HDL and lipid free ApoA-I are transported through endothelial 
cell layers by regulated processes: ApoA-I is first lipidated by the ATP binding cassette transporter $(\mathrm{ABC}) \mathrm{A} 1$ prior to its transport through the endothelial cells [11]. Transendotelial HDL transport is modulated by ABCG1, scavenger receptor B I (SR-BI), endothelial lipase (EL) [6], and the ectopic betaATPase/purinergic receptor axis [12]. None of these proteins is a good candidate to directly mediate holoparticle uptake. They appear rather to indirectly modulate transendothelial transport of HDL by signalling or altering the structure of HDL.

In this study we applied microscopic techniques to better characterize the transendothelial transport of HDL. 


\section{Materials and Methods}

\section{Cell culture}

Primary Bovine Aortic Endothelial cells (EC) were cultured in Dulbecco's modified eagle's medium (DMEM) with $5 \%$ fetal bovine serum at $37{ }^{\circ} \mathrm{C}$ in $5 \%$ $\mathrm{CO}_{2}$ and passaged every $7-8$ days $1: 1 . \mathrm{A} 10 \mathrm{~cm}$ cell culture treated petri dish was populated with $2.5-3 \times 10^{6}$ cells.

\section{Preparation and labeling of lipoproteins and marker proteins}

HDL and LDL were isolated from normolipidemic human plasma (blood bank of cantonal hospital Schaffhausen) by sequential ultracentrifugation following the protocol described by Tong et al [13] and stored at $4{ }^{\circ} \mathrm{C}$. Lipid free ApoA-I was generated by delipidation of HDL by ethanol-ether [14] and purification over an anion exchanger column (Mono-Q) on the Äkta FPLC (GE Healthcare). Qualitative purity control was performed by SDS-PAGE, coomassie-staining verifying the HDL and ApoA-I fractions to be void of visible albumin and $A p o B(L D L)$ bands.

Lipoproteins were labeled with $1.4 \mathrm{~nm}$ nanogold (Nanoprobes USA) (Au-HDL) or fluorescent Atto dyes Atto488, Atto594, Atto655, obtained as succimidylester (Atto-Tec, Germany) and set to $\mathrm{pH} 8.0$ by $1 \mathrm{M} \mathrm{NaHCO}_{3}$ to a final concentration of $0.1 \mathrm{M}$. The dye to lipoprotein (molar) ratio was $5: 1$, the lipoprotein concentration in the labeling reaction was $6 \mathrm{~g} / \mathrm{l}$. The reaction was performed in the dark at room temperature for 2 hours. The labeled lipoproteins were separated from free label by gel filtration chromatography (NAP5 and PD10 columns GE). Bovine Serum Albumin (Sigma-Aldrich, CH) 
and Transferrin (Invitrogen) were labeled with fluorescent dyes as described for lipoproteins.

\section{Binding and uptake studies by fluorescence light (FLM) and electron microscopy (EM)}

For both fluorescence light microscopy and electron microscopy experiments, 10 '000 cells were seeded 48-96 hours before the experiments either onto collagen I coated transwell inserts of 24 well dishes ( 0.4 um low density, BD) or round $\mathrm{Nr} 1.0$ glass coverslips of $12 \mathrm{~mm}$ diameter (Metzler Glass / Thermo Fisher). To analyze their uptake and subcellular distribution, the labeled lipoproteins were added to the cells for 30 minutes at $37^{\circ} \mathrm{C}$ in $5 \% \mathrm{CO} 2$ if not otherwise indicated, followed by rinsing with ice cold PBS++ (PBS 7.4 with 1 $\mathrm{mM} \mathrm{CaCl}_{2}$ and $0.1 \mathrm{mM} \mathrm{MgCl}_{2}$ ). For binding experiments, the cells were incubated at $4^{\circ} \mathrm{C}$ in a humidified closed box. Subsequently, cells were either chemically fixed for fluorescence microscopy or high pressure frozen (HPF) for EM analysis.

\section{Fixation for FLM}

Chemical fixation for FLM was performed with 3.75\% formaldehyde in PBS at rt for 20-30 minutes for monolayers of cells. The free fixative was then quenched by a brief wash with $0.5 \mathrm{M}$ TrisHCL 8.0 followed by two washes with PBS. Then either additional stainings were performed or the sample was embedded directly for microscopy.

\section{Indirect Immunostainings for FLM}

Coverslips or transwell inserts with fixed cells were preincubated with $0.1 \%$ saponin in PBS at $\mathrm{pH} 7.4$ at room temperature for 15 minutes for antibody 
staining . The primary antibody was incubated for one hour at rt or $16 \mathrm{~h}$ at 4 ${ }^{\circ} \mathrm{C}$, the cells were then washed tree times with PBS and then incubated with the secondary antibody for $30-45$ minutes at $r$ in the dark. Antibody dilutions were made in PBS with $1 \%$ Donkey Serum (Sigma Aldrich) and $5 \%$ BSA. Optimal antibody titres were determined beforehand by testing different dilutions ranging from 1:200 to 1:10'000. Subsequently, the coverslips were directly mounted upside down on glass slides with ProLong Gold antifade solution (Life technologies, USA). Transwells were cut out with a scalpel Nr11 from the bottom side and placed with the cells upwards between a glass slide and a 25x50 mm Nr 1.0 coverslip (Metzler Glass / Thermo Fisher) with a drop of ProLong Gold inbetween.

Images were acquired on a Zeiss Axiovert 200M with a Plan-Apochromat 40x/1.4 and Hamamatsu ORCA-ER EMCCD camera (C4742) resulting in a pixel size of $0.1577 \mu \mathrm{m}$. To be able to quantitatively compare conditions, fixed exposure times were used of 10 to $800 \mathrm{~ms}$ to have the signal histogram of the (positive) control in the middle of the 12-bit sensitivity range of the chip and this exposure was kept throughout the acquisition session.

\section{Image processing and Quantification}

To quantify the HDL taken up by ECs, we segmented the cells in the monolayer and then measured fluorescence intensities per cell. Segmentation was performed using the open source FIJI is just ImageJ (FIJI) software. First the nucleus was isolated in the DAPI channel by the Otsu thresholding method. Based on the nucleus and using the Voronoi algorithm the cells were approximated. For quantification, only the cells, which were completely in the 
field of view, were considered. The mean fluorescence signal per cell was calculated for at least 200 cells per condition and experiment.

When co-localization was neither complete nor absent upon visual inspection, partial co-localization was quantified by using FIJl's Coloc2 plugin to calculate the Pearson's correlation coefficient $\rho$.

\section{High pressure freezing (HPF) and freeze substitution fixation (FSF) for}

\section{EM}

The membrane of the transwell containing a monolayer of endothelial cells was punched out with a biopsy-punch of $5 \mathrm{~mm}$ diameter. The isolated membrane disc was carefully inserted into a $6 \mathrm{~mm}$ aluminium specimen carrier with an indentation of $100 \mu \mathrm{m}$, sandwiched with a flat carrier wetted with 1-hexadecene (Sigma Aldrich, $\mathrm{CH}$ ) and immediately high pressure frozen using an automated Leica EM HPM100 high pressure freezing machine (Leica Microsystems, Vienna, Austria). Samples were stored in liquid nitrogen until further processing.

HPF frozen discs containing membranes with cell monolayers were transferred to $2 \mathrm{ml}$ safe-lock Eppendorf tubes containing anhydrous acetone with $1 \%$ OsO4 at $-90{ }^{\circ} \mathrm{C}$. Substitution was performed in an automated substitution machine (Leica EM AFS) at $-90^{\circ} \mathrm{C}$ for $7 \mathrm{~h},-60^{\circ} \mathrm{C}$ for $6 \mathrm{~h},-30^{\circ} \mathrm{C}$ for $5 \mathrm{~h}$ and at $0{ }^{\circ} \mathrm{C}$ for $1 \mathrm{~h}$ with transition gradients of $30^{\circ} \mathrm{C}$ per hour.

\section{Silver enhancement}

The reagents (Sigma Aldrich, $\mathrm{CH}$ ) were prepared in individual tubes as saturated solutions of hydroquinone, malic acid, and silver nitrate in anhydrous acetone at room temperature. Arabic gum was dispersed in 
anhydrous acetone and shaken for one hour at room temperature. The Arabic gum dispersion was used to protect the colloid from aggregation by inhibiting the self-nucleation of silver.

After slowly cooling the saturated solutions to $0^{\circ} \mathrm{C}$ during one hour, equal parts of all saturated solutions and the Arabic gum suspension were mixed at $0{ }^{\circ} \mathrm{C}$. The mixture was centrifuged at $300 \mathrm{~g}$ for $5 \mathrm{~min}$ at $4{ }^{\circ} \mathrm{C}$ to remove any precipitates and was always freshly prepared before use.

After FSF the samples were washed three times with anhydrous acetone and the enhancement mixture was added to the reaction tubes for 30 minutes. Afterwards, the treated samples were washed again three times with anhydrous acetone before embedding in epoxy resin. All steps were performed at $0{ }^{\circ} \mathrm{C}$ on ice water.

\section{Embedding and preparation for TEM and EM image acquisition}

Samples in anhydrous acetone were embedded in Epon/Araldite (EA) essentially as described by Hohenberg et al. $[15,16]$ by incubating the samples in $33 \%$ and $66 \%$ EA in acetone for $8 \mathrm{~h}$ each prior to transfer in 100 $\%$ EA and polymerization at $60{ }^{\circ} \mathrm{C}$ for 40 hours.

Ultrathin cross sections of cells of $50 \mathrm{~nm}$ were cut with a $45^{\circ}$ diamond knife (Diatome) using an ultramicrotome (Reichert) and put on Formvar coated single slot grids (Ted Pella inc. USA).

Images were acquired with a Philips CM100 or a FEI Tecnai G2 Spirit transmission electron microscope ( $\mathrm{FEI}$, Eindhoven, The Netherlands) at an acceleration voltage of $80 \mathrm{kV}$ or $120 \mathrm{kV}$ using a Gatan Orius 1000 camera (Gatan Inc. USA). 


\section{Results}

\section{Kinetics of intracellular HDL transport}

To monitor the uptake and transport of HDL over time, we added fluorescently labelled $\mathrm{HDL}$ to monolayers of $\mathrm{ECs}$ at $37^{\circ} \mathrm{C}$ for different time intervals ranging from 5 minute to 60 minutes. HDL was detected within the cells after about 5 minutes and the signal increased with prolonged incubation. At different time points, HDL appeared to reside in different compartments of the cells. The pattern of HDL fluorescence after one hour is clearly different from the earlier time points, however they have in common that the HDL positive vesicles are found closely to and primarily on one side of the nucleus. (Figure 1 A-D). To verify the trafficking through different vesicles, we performed pulse chase experiments. First a green labelled HDL was incubated overnight with ECs for internalisation. Then a red-labelled HDL was added for different time points ranging from 5 min to 4 hours (Figure 2). After 2 hours, the newly added HDL started colocalising with that HDL that was pre-incubated over night with the ECs.

\section{Co-localisation of HDL with ApoA-I and other plasma proteins}

To test if lipid free ApoA-I and HDL are trafficked through the same pathway, we simultaneously incubated ECs with 594-HDL and 488-ApoA-I and found complete co-localization ( $\rho=0.77$, Figure 3 A-C). Interestingly we found 594HDL partially co-localized with 488-LDL, $(\rho=0.56$, Figure $3 \mathrm{D}-\mathrm{F})$. 
We observed also partial co-localization of 488-HDL with 594-Albumin ( $\rho=$ 0.62, Figure 4 A-C) and 594-transferrin ( $\rho=0.47$, Figure $4 \mathrm{D}-\mathrm{F})$, which are known to be internalized by clathrin- or caveolin-dependent endocytosis. We did, however, not observe any direct co-localization of 488-HDL with clathrin or caveolin 1 during 30 minutes of incubation. (Figure $4 \mathrm{H}$ and I).

\section{HDL is not found in typical trafficking organelles}

We next explored the co-localization of HDL with markers of early or late endosomes (Rab5 and Rab7, respectively) (Figure $5 \mathrm{~A}$ and B) and endosome-trans golgi network trafficking (Rab9 and Syntaxin6) (Figure $5 \mathrm{C}$ and D). ECs were incubated with $488-\mathrm{HDL}$ or $594-\mathrm{HDL}$ for 30 minutes and after fixation, the cells were stained against the proteins described above. Unfortunately none of the vesicle markers we used appeared to co-localize with the red labeled 594-HDL indicating that HDL passes very quickly through endosomes to end up in vesicles that are Rab5 and/or Rab7 negative. Lack of co-localization of 594-HDL with either Syntaxin 6 or Rab9 indicate that HDL is transported neither to the plasma membrane nor into the trans golgi network. The green labeled 488-HDL was co-localized with neither lysosomes (marked by Lysotracker DND 99) nor late endosomes (Rab7 positive vesicles) (Figure $5 \mathrm{E})$ nor recycling endosomes (Rab11a). (Figure $5 \mathrm{~F}$ ). Further we also tested for colocalisation with the endoplasmatic reticulum (Calnexin, Figure $5 \mathrm{G}$ ) and the Golgi (58kD Golgi Protein, Figure $5 \mathrm{H}$ ) and could not detect any colocalisation.

Next we applied electron microscopy to ECs, which were incubated with AuHDL. It was found mainly localized in large vesicles that were filled with additional electron dense material (Figure 6A and 6B) and thereby resembled 
multivesicular bodies (MVB's) or autophagosomes. However, neither the MVB markers CHMP4b, 6, 7 and CD63 (figures 6C, 6D, 6E and 6F, respectively) nor the autophagy marker LC3 (figure 6G) were found colocalized with fluorescent HDL.

HDL crosses the retroendocytotic pathway taken by Lectins To follow the intracellular route of HDL containing vesicles, we used 488-HDL with fluorescently labeled lectin wheat germ agglutinin (594-WGA) to label cell surface $\mathrm{N}$-acetyl-D-glucosamines of extracellular exposed proteins which are subject to endocytosis and recycling and therefore label a broad range of vesicles being taken up by ECs. ECs were incubated for 20 minutes with 594WGA and then for different time intervals with 488-HDL. After 15 min 488HDL and 594-WGA co-localized (Figure 7A). The co-localization diminishes over time (Figure 7B, $30 \mathrm{~min}$ ) and is entirely lost after $60 \mathrm{~min}$ (Figure 7C).

\section{HDL uptake and intracellular trafficking is cytosol and dynamin dependent}

Cytoskeleton disruption with cytochalasin D or colchicine or the dynamin blocker dynasore resulted in reduced 594-HDL uptake into ECs (Figure 8). Interference with actin by cytochalasin D led to the smallest decrease (15.7\%) and caused no qualitative shift of HDL-positive vesicles from perinuclear to peripheral compartments (Figure 8 B,D). Tubulin disruption by colchicine caused a significantly (t-test $\mathrm{p}=0.0005$ ) more pronounced decrease in 594HDL uptake by $35.5 \%$. Moreover, 594-HDL remained in small intracellular 
peripheral vesicles (Figure 8 C). A similar 35.3\% decrease of 594-HDL uptake was detected after incubation of ECs with $120 \mu \mathrm{M}$ dynasore for 20 minutes at $37^{\circ} \mathrm{C}$, (Figure $\left.8 \mathrm{D}\right)$.

\section{HDL is not taken up via fluid phase by endothelial cells}

To test for HDL uptake by fluid phase, we treated ECs with Amiloride and EIPA, two inhibitors of $\mathrm{Na} / \mathrm{H}$ channels needed for induction of fluid phase trafficking by localized pH-shift. In either condition 655-HDL uptake was almost normal indicating that HDL uptake is not driven by fluid phase (Figure 9 A-C). Quantification shows only slight but not significant reduction in mean fluorescence per cell (10\%). To corroborate this finding, ECs were coincubated with fl-HDL together with fluorescently labeled $40 \mathrm{kDa}$ Dextran, a marker for fluid phase uptake. However, no co-localization was visible (Figure 9 E-G). 


\section{Discussion}

To elucidate the itinerary of ApoA-I and HDL through ECs, we investigated the cellular localization of fluorescently or gold labelled ApoA-I and HDL. HDL as well as initially lipid-free ApoA-I are taken up by ECs. After 30 minutes HDL co-localizes with ApoA-I independently of whether the two were added to EC's in parallel or sequentially. This finding supports our previously suggested model that lipid-free ApoA-I is lipidated by ABCA1 to be internalized by the same route as artificial discoidal or native spherical HDL[17].. Based on this finding we limited our subsequent experiments to HDL.

Our time kinetic (figure 1) and pulse-chase experiments (figure 2) indicate that HDL are trafficked through different vesicles until they accumulate after 2 hours in a common sink which probably corresponds to the large vesicles in which we retrieved Au-HDL by electron microscopy. Since our previous biochemical experiments indicate that $\mathrm{HDL}$ is at least partially resecreted, our the findings of our microscopic studies can be explained by two models:

1. HDL is trafficked through ECs by two parallel routes, one very fast leading to direct resecretion of HDL and one much slower leading to the accumulation of HDL within MVBs. Such a model would be in agreement with the calculations of Michel et al. [18]

2. HDL is trafficked through one route and HDL accumulates in MVB's if endocytosis is faster than exocytosis.

We observed some co-localization of HDL with albumin, transferrin and LDL but not with any typical marker of organelles and vesicles. Since we used epifluorescence and since endothelial cells are relatively flat, it is unprobable that we missed any positive co-localization. Our experiments using 
pharmacological inhibitors indicate that HDL uptake depends on intact cytoskeleton and does not involve fluid phase uptake. HDL hence appears to be trafficked by an atypical endocytic route through ECs.

\section{Role of clathrin, caveolae and fluid phase for HDL uptake}

HDL was partially co-localized with transferrin, albumin, and LDL. Like HDL all are internalized and re-secreted by endothelial cells to supply subendothelial cells with their cargo, notably fatty acids, iron, and cholesterol, respectively. Albumin uptake depends on caveolin 1 and involves gp60 as the receptor $[19,20]$. Transferrin interacts with the transferrin receptor and was reported to pass the endothelium by both clathrin dependent and independent pathways [21-25].. The canonical internalization of native LDL by LDL receptors relies on the formation of coated pits and hence clathrin. However, the reduced uptake of LDL by endothelial cells from caveolin 1 knock-out mice indicate that LDL can also be internalized by non-clathrin dependent pathways [26]. At $37^{\circ} \mathrm{C}$ we observed no co-localization of $\mathrm{HDL}$ with clathrin and Caveolin 1. In conjunction with the lack of complete co-localization of HDL with albumin, transferrin, native LDL or AcLDL, this indicates that $H D L$ is internalized by ECs through a non-clathrin- and non-caveolin-dependent endocytic pathway. We suspected that HDL is taken up by fluid phase. The underlying pinocytosis does not require any defined receptor-ligandinteraction but is a bulk transport regulated independently of dynamin 2, clathrin and caveolin in epithelial cells [27]. For example, viruses are known to 
be internalized by pinocytosis $[28,29]$. However, blocking of $\mathrm{Na} / \mathrm{H}$-channels essential for any fluid phase transport by Amiloride and/or EIPA even at high concentrations did not change HDL uptake. Neither did we find any colocalization of fl-HDL with fluorescently labelled $40 \mathrm{kDa}$ Dextran, which is taken up by cells through fluid phase endocytosis. By contrast, dynasore [30], an inhibitor of dynamin dependent vesicle formation significantly reduced HDL uptake. These findings indicate that HDL is not taken up by fluid phase.

\section{HDL appear neither in recycling endosomes nor in lysosomes}

HDL failed to co-localize with either Rab5, a protein found on most endocytic endosomes, or Rab7, a marker for late endosomes. Within time intervals shorter than 30 minutes, HDL positive vesicles were too faint to indicate any co-localization. This suggests that HDL passes the endosomes very quickly or in low amounts to accumulate in vesicles, or other compartments, which are rab5 and rab7 negative. HDL did neither colocalize with Rab11a which is a marker for recycling vesicles transporting membrane and material coming from the apical surface back to the apical membrane. In agreement with the trans-endothelial apical-to-basolateral transport that we observed by biochemical experiments [6] it is hence very unlikely that $\mathrm{HDL}$ travels through the recycling route..

Excluding any significant transport of HDL into lysosomes, we found no colocalization of fl-HDL with Lysotracker DND 99 and Rab7. In agreement with this biochemical turnover studies from our lab indicate that there is almost no degradation of $\mathrm{HDL}$ proteins within ECs [6]. and 
Interestingly, HDL co-localised partially with LDL and transferrin which in many cells are transported to lysosomes for supplying the cells with cholesterol and iron, respectively. Endothelial cells were found to deviate from this canonical pathway: Only a part of LDL internalized by ECs is transported to lysosomes and degraded together with its receptor [31]. A considerable part of LDL is rather transcytosed by ECs through caveolin 1 containing vesicles [32]. It is hence well possible that HDL, LDL and transferrin share common transendothelial trafficking pathways which avoid lysosomal degradation.

\section{Intracellular accumulation of HDL in large vesicles}

As after 30 minutes intracellular 488-HDL clusters close to one side of the nucleus, we suspected that it is transcytosed through the transgolgi network (TGN). However, staining the cells incubated with fl-HDL for Golgi 58kDa protein and calnexin as markers for golgi and endoplasmatic reticulum (ER), respectively, revealed no co-localization. Neither did we see any colocalization with rab9 and syntaxin6, both being involved in the trafficking to the TGN and golgi $[33,34]$. This indicates, that HDL is localized in vesicles in close proximity to TGN, golgi or ER-golgi intermediate compartment (ERGIC) [35] but it is not directly crossing these metabolically active organelles of ECs. The sorting of these vesicles appears to involve tubulin rather than actin since destruction of microtubules by colchicine but not the destabilization of f-actin by cytochalasin $D$ interfered with the accumulation of HDL in perinuclear compartments and restrained HDL in the periphery. 
We assume that these perinuclear HDL containing vesicles correspond to the large vesicles in which our electron microscopy studies localized the majority of Au-HDL. By the content of electron dense material these vesicles resembled MVBs which are the main sorting stations of vesicular traffic, or autophagosomes. However, no marker of these specialized vesicles was colocalized with HDL. Interestingly, also other cells appear to store internalized HDL in intracellular organelles, for example hepatocytes [36] , macrophages [37], enterocytes [38,39], or even parasitic protozoans like trypanosomes. In macrophages and hepatocytes internalized HDL has been proposed to mobilize cholesterol and ApoE, respectively, by retroendoctosis [40,41]. In enterocytes HDL was postulated to interfere with NFאB activation and hence inflammatory response by trapping $I_{\kappa} B$ in autophagosomes [42]. The internalization of HDL into trypanosomes provides ApoL1, which kills the protozoa by lysosomal swelling [43]. The intracellular residence of a considerable proportion of $\mathrm{HDL}$ in ECs over a rather long period of time without being degraded and secreted raises the question whether this phenomenon also contributes to the protective effects of $\mathrm{HDL}$ to the endothelium [5,44].

\section{Conclusion}

By using fluorescence and electron microscopy we support our previous biochemical data that HDL is internalized by endothelial cells without undergoing significant degradation. Internalisation and trafficking involves dynamin and the cytoskeleton. Interestingly, we found HDL not co-localized 
with any marker of classical endocytic pathways that are clathrin coated pits, caveolae, or fluid phase. Surprisingly, a substantial proportion of internalized HDL was found to accumulate in atypical large vesicles either for storage before basolateral release or for the exertion of as yet unknown functions.

\section{Acknowledgements}

We are thanking Silvija Radosavljevic, Bruno Guhl, Ursula Lüthi, Therese Bruggmann and Gery Barmettler for their technical assistance.

This work was supported by grants from the Swiss National Science Foundation (31003A-130836/I), the COST program of the Federal Department of Economic Affairs, Education and Research (project C12.0122), and the $7^{\text {th }}$ Framework Program of the European Commission ("TransCard"; project number 603091). AvE, LR, and DP are members of the European COST action BM904 (HDLnet). 


\section{References}

[1] J. a Didonato, Y. Huang, K.S. Aulak, O. Even-Or, G. Gerstenecker, V. Gogonea, et al., Function and distribution of apolipoprotein A1 in the artery wall are markedly distinct from those in plasma., Circulation. 128 (2013) 1644-55. doi:10.1161/CIRCULATIONAHA.113.002624.

[2] E. Di Angelantonio, N. Sarwar, P. Perry, S. Kaptoge, K.K. Ray, A. Thompson, et al., Major lipids, apolipoproteins, and risk of vascular disease., JAMA. 302 (2009) 1993-2000. doi:10.1001/jama.2009.1619.

[3] K.R. Norum, T. Berg, P. Helgerud, C.A. Drevon, Transport of cholesterol., Physiol. Rev. 63 (1983) 1343-419. http://www.ncbi.nlm.nih.gov/pubmed/6361811 (accessed February 6, 2014).

[4] G.H. Rothblat, M. Bamberger, M.C. Phillips, Reverse cholesterol transport., Methods Enzymol. 129 (1986) 628-44.

http://www.ncbi.nlm.nih.gov/pubmed/3523160 (accessed February 6, 2014).

[5] W. Annema, A. von Eckardstein, High-density lipoproteins. Multifunctional but vulnerable protections from atherosclerosis., Circ. J. 77 (2013) 2432-48. http://www.ncbi.nlm.nih.gov/pubmed/24067275 (accessed July 15, 2014).

[6] L. Rohrer, P.M. Ohnsorg, M. Lehner, F. Landolt, F. Rinninger, A. von Eckardstein, High-Density Lipoprotein Transport Through Aortic Endothelial Cells Involves Scavenger Receptor BI and ATP-Binding Cassette Transporter G1, Circ. Res. 104 (2009) 1142-1150. doi:10.1161/CIRCRESAHA.108.190587.

[7] H.Y. Lim, C.H. Thiam, K.P. Yeo, R. Bisoendial, C.S. Hii, K.C.Y. McGrath, et al., Lymphatic vessels are essential for the removal of cholesterol from peripheral tissues by SR-BI-mediated transport of HDL., Cell Metab. 17 (2013) 671-84. doi:10.1016/j.cmet.2013.04.002.

[8] C. Martel, W. Li, B. Fulp, A.M. Platt, E.L. Gautier, M. Westerterp, et al., Lymphatic vasculature mediates macrophage reverse cholesterol transport in mice, 123 (2013) 1571-1579. doi:10.1172/JCI63685DS1.

[9] E.E. Ebong, F.P. Macaluso, D.C. Spray, J.M. Tarbell, Imaging the endothelial glycocalyx in vitro by rapid freezing/freeze substitution transmission electron microscopy., Arterioscler. Thromb. Vasc. Biol. 31 (2011) 1908-15. doi:10.1161/ATVBAHA.111.225268. 
[10] J.R. Levick, C.C. Michel, Microvascular fluid exchange and the revised Starling principle., Cardiovasc. Res. 87 (2010) 198-210. doi:10.1093/cvr/cvq062.

[11] C. Cavelier, L. Rohrer, A. von Eckardstein, ATP-Binding cassette transporter A1 modulates apolipoprotein A-I transcytosis through aortic endothelial cells., Circ. Res. 99 (2006) 1060-6. doi:10.1161/01.RES.0000250567.17569.b3.

[12] C. Cavelier, P.M. Ohnsorg, L. Rohrer, A. von Eckardstein, The $\beta$-chain of cell surface $F(0) F(1)$ ATPase modulates apoA-I and HDL transcytosis through aortic endothelial cells., Arterioscler. Thromb. Vasc. Biol. 32 (2012) 131-9. doi:10.1161/ATVBAHA.111.238063.

[13] H. Tong, H.R. Knapp, M. VanRollins, A low temperature flotation method to rapidly isolate lipoproteins from plasma., J. Lipid Res. 39 (1998) 1696-704. http://www.ncbi.nlm.nih.gov/pubmed/9717731.

[14] W. V Brown, R.I. Levy, D.S. Fredrickson, Studies of the proteins in human plasma very low density lipoproteins., J. Biol. Chem. 244 (1969) 5687-94. http://www.ncbi.nlm.nih.gov/pubmed/4981584 (accessed January 27, 2014).

[15] J.H. Luft, Improvements in epoxy resin embedding methods, J. Biophys. Biochem. Cytol. 9 (1961) 409-414.

[16] H. Hohenberg, K. Mannweiler, M. Müller, High-pressure freezing of cell suspensions in cellulose capillary tubes, J. Microsc. 175 (1994) 34-43. doi:10.1111/j.1365-2818.1994.tb04785.x.

[17] P.M. Ohnsorg, L. Rohrer, D. Perisa, A. Kateifides, A. Chroni, D. Kardassis, et al., Carboxyl terminus of apolipoprotein A-I (ApoA-I) is necessary for the transport of lipid-free ApoA-I but not prelipidated ApoA-I particles through aortic endothelial cells., J. Biol. Chem. 286 (2011) 7744-54. doi:10.1074/jbc.M110.193524.

[18] C.C. Michel, M.N. Nanjee, W.L. Olszewski, N.E. Miller, LDL and HDL transfer rates across peripheral microvascular endothelium agree with those predicted for passive ultrafiltration in humans, J. Lipid Res. 56 (2015) 122-128. doi:10.1194/jlr.M055053.

[19] J.S. Geoffroy, R.P. Becker, Endocytosis by endothelial phagocytes: uptake of bovine serum albumin-gold conjugates in bone marrow., J. Ultrastruct. Res. 89 (1984) 223-39.

http://www.ncbi.nlm.nih.gov/pubmed/6544888.

[20] S.M. Vogel, R.D. Minshall, M. Pilipović, C. Tiruppathi, A.B. Malik, Albumin uptake and transcytosis in endothelial cells in vivo induced by albumin-binding protein., Am. J. Physiol. Lung Cell. Mol. Physiol. 281 
(2001) L1512-22. http://www.ncbi.nlm.nih.gov/pubmed/11704548 (accessed November 1, 2013).

[21] T. Kishimoto, M. Tavassoli, Transendothelial transport (transcytosis) of iron-transferrin complex in the rat liver., Am. J. Anat. 178 (1987) 241-9. doi:10.1002/aja.1001780305.

[22] J.B. Fishman, J.B. Rubin, J. V Handrahan, J.R. Connor, R.E. Fine, Receptor-mediated transcytosis of transferrin across the blood-brain barrier., J. Neurosci. Res. 18 (1987) 299-304.

doi:10.1002/jnr.490180206.

[23] R.L. Roberts, R.E. Fine, a Sandra, Receptor-mediated endocytosis of transferrin at the blood-brain barrier., J. Cell Sci. 104 ( Pt 2 (1993) 52132. http://www.ncbi.nlm.nih.gov/pubmed/8505377.

[24] R.L. Roberts, A. Sandra, Transport of transferrin across the bloodthymus barrier in young rats., Tissue Cell. 26 (1994) 757-66.

http://www.ncbi.nlm.nih.gov/pubmed/9437249 (accessed January 28, 2014).

[25] T. Tsuji, H. Yoshitomi, J. Usukura, Endocytic mechanism of transferrinconjugated nanoparticles and the effects of their size and ligand number on the efficiency of drug delivery., Reprod. Syst. Sex. Disord. 62 (2013) 341-52. doi:10.1093/jmicro/dfs080.

[26] P.G. Frank, S. Pavlides, M.W.-C. Cheung, K. Daumer, M.P. Lisanti, Role of caveolin-1 in the regulation of lipoprotein metabolism., Am. J. Physiol. Cell Physiol. 295 (2008) C242-C248.

doi:10.1152/ajpcell.00185.2008.

[27] H. Cao, J. Chen, M. Awoniyi, J.R. Henley, M. a McNiven, Dynamin 2 mediates fluid-phase micropinocytosis in epithelial cells., J. Cell Sci. 120 (2007) 4167-77. doi:10.1242/jcs.010686.

[28] J. Mercer, A. Helenius, Virus entry by macropinocytosis., Nat. Cell Biol. 11 (2009) 510-20. doi:10.1038/ncb0509-510.

[29] J. Mercer, A. Helenius, Gulping rather than sipping: macropinocytosis as a way of virus entry., Curr. Opin. Microbiol. 15 (2012) 490-9. doi:10.1016/j.mib.2012.05.016.

[30] E. Macia, M. Ehrlich, R. Massol, E. Boucrot, C. Brunner, T. Kirchhausen, Dynasore, a cell-permeable inhibitor of dynamin., Dev. Cell. 10 (2006) 839-50. doi:10.1016/j.devcel.2006.04.002.

[31] E. Vasile, M. Simionescu, N. Simionescu, Visualization of the binding, endocytosis, and transcytosis of low-density lipoprotein in the arterial endothelium in situ., J. Cell Biol. 96 (1983) 1677-89. 
http://www.pubmedcentral.nih.gov/articlerender.fcgi?artid=2112465\&too I=pmcentrez\&rendertype=abstract.

[32] F. Bian, X. Yang, F. Zhou, P.-H. Wu, S. Xing, G. Xu, et al., CRP promotes atherosclerosis by increasing LDL transcytosis across endothelial cells., Br. J. Pharmacol. (2014). doi:10.1111/bph.12616.

[33] J.B. Bock, J. Klumperman, S. Davanger, R.H. Scheller, Syntaxin 6 functions in trans-Golgi network vesicle trafficking., Mol. Biol. Cell. 8 (1997) 1261-1271. doi:10.1091/mbc.8.7.1261.

[34] E.L. Ng, B.Q. Gan, F. Ng, B.L. Tang, Rab GTPases regulating receptor trafficking at the late endosome-lysosome membranes., Cell Biochem. Funct. 30 (2012) 515-23. doi:10.1002/cbf.2827.

[35] C. Appenzeller-Herzog, H.-P. Hauri, The ER-Golgi intermediate compartment (ERGIC): in search of its identity and function., J. Cell Sci. 119 (2006) 2173-83. doi:10.1242/jcs.03019.

[36] C. Röhrl, T. a Pagler, W. Strobl, A. Ellinger, J. Neumüller, M. Pavelka, et al., Characterization of endocytic compartments after holo-high density lipoprotein particle uptake in HepG2 cells., Histochem. Cell Biol. 133 (2010) 261-72. doi:10.1007/s00418-009-0672-3.

[37] G. Schmitz, M. Grandl, The molecular mechanisms of HDL and associated vesicular trafficking mechanisms to mediate cellular lipid homeostasis., Arterioscler. Thromb. Vasc. Biol. 29 (2009) 1718-22. doi:10.1161/ATVBAHA.108.179507.

[38] G. Rogler, G. Herold, C. Fahr, M. Fahr, D. Rogler, F.M. Reimann, et al., High-density lipoprotein 3 retroendocytosis: a new lipoprotein pathway in the enterocyte (Caco-2)., Gastroenterology. 103 (1992) 469-480.

[39] G. Rogler, E. Aschenbrenner, V. Gross, E.F. Stange, J. Schölmerich, Intracellular transport of high-density lipoprotein 3 in intestinal epithelial cells (Caco-2) is tubulin associated, Digestion. 61 (2000) 47-58. doi:10.1159/000007735.

[40] G. Schmitz, H. Robenek, U. Lohmann, G. Assmann, Interaction of high density lipoproteins with cholesteryl ester-laden macrophages: biochemical and morphological characterization of cell surface receptor binding, endocytosis and resecretion of high density lipoproteins by macrophages., EMBO J. 4 (1985) 613-622.

[41] J. Heeren, T. Grewal, A. Laatsch, D. Rottke, F. Rinninger, C. Enrich, et al., Recycling of apoprotein $E$ is associated with cholesterol efflux and high density lipoprotein internalization, J. Biol. Chem. 278 (2003) 14370-14378. doi:10.1074/jbc.M209006200. 
[42] R. Gerster, J.J. Eloranta, M. Hausmann, P. a. Ruiz, J. Cosin-Roger, A. Terhalle, et al., Anti-inflammatory Function of High-Density Lipoproteins via Autophagy of IkB Kinase, C. Cell. Mol. Gastroenterol. Hepatol. 1 (2015) 171-187. doi:10.1016/j.jcmgh.2014.12.006.

[43] J.M. Harrington, S. Howell, S.L. Hajduk, Membrane permeabilization by trypanosome lytic factor, a cytolytic human high density lipoprotein, J. Biol. Chem. 284 (2009) 13505-13512. doi:10.1074/jbc.M900151200.

[44] C. Mineo, P.W. Shaul, Regulation of signal transduction by HDL., J. Lipid Res. 54 (2013) 2315-24. doi:10.1194/jlr.R039479. 


\section{Figure Legends}

Figure 1: Time dependent uptake of fluorescent HDL by endothelial cells. A monolayer of ECs was incubated for (A) $5 \mathrm{~min},(B) 15 \mathrm{~min},(\mathbf{C}) 30$ min and (D) $60 \mathrm{~min}$ at $37^{\circ}$ with $50 \mathrm{ug} / \mathrm{ml}$ 594-HDL, fixed with $3.75 \%$ formaldehyde and counter-stained with DAPI for nuclear staining. The longer 594-HDL is incubated with ECs, the more can be detected intracellularly. white bar $=20 \mu \mathrm{m}$

Figure 2: Characterization of endothelial HDL trafficking by a pulsechase experiment . First, ECs were incubated over night with green 488$\mathrm{HDL}$, which was then washed away before red 594-HDL was added to the cells. The cells were fixed at different times after the second incubation of 5 $\min (A), 10 \min (B), 15 \min (C), 30 \min (D), 60 \min (E) 120 \min (F), 180 \min$ (G) and 240 min $(H)$. After two and four hours $(G, H)$ the chasing green HDL was found colocalising with the pulsed red HDL.

Figure 3: Co-localization of HDL with apoA-I (A-C) and native LDL (D-F) A monolayer of ECs was incubated for $30 \mathrm{~min}$ at $37^{\circ}$ with $50 \mu \mathrm{g} / \mathrm{ml}$ of each 488-ApoA-I (A) and 594-HDL (B), fixed with $3.75 \%$ formaldehyde and counter-stained with DAPI for nuclear staining. The merge of $\mathbf{A}$ and $\mathbf{B}$ shows uniform co-localization of ApoA-I and HDL (C). A monolayer of ECs was incubated for $30 \mathrm{~min}$ at $37^{\circ}$ with $50 \mu \mathrm{g} / \mathrm{ml} 655-\mathrm{HDL}$ (D) or, $20 \mu \mathrm{g} / \mathrm{ml} 488-\mathrm{LDL}$ (E), fixed with $3.75 \%$ formaldehyde and counter-stained with DAPI for nuclear 
staining. The merge of $\mathbf{D}$ with $\mathbf{E}$ shows that HDL partial co-localizes with LDL (F). white bar $=20 \mu \mathrm{m}$

Figure 4: Co-,localisation of HDL with transferrin (A-C), albumin (D-F) within endothelial cells

Monolayers of ECs were incubated for $30 \mathrm{~min}$ at $37^{\circ}$ with $50 \mu \mathrm{g} / \mathrm{ml}$ FITC-HDL (A, D and G) and $5 \mu \mathrm{g} / \mathrm{ml}$ 594-Transferrin (B) or $10 \mu \mathrm{g} / \mathrm{ml}$ 546-Albumin (E), fixed with $3.75 \%$ formaldehyde and counter-stained with DAPI for nuclear staining. The merges of $A$ with $B(\mathbf{C})$ and $D$ with $E(\mathbf{F})$ show partial colocalization of HDL with Transferrin (C) and albumin (F), respectively. Further stainings for Clathrin $(\mathbf{H})$ and Caveolin 1 (I) showed also no colocalisation with these organelles with HDL. white bar $=20 \mu \mathrm{m}$

Figure 5: Co-localization of HDL with different vesicles and organelles of endothelial cells.

EC Monolayers were incubated for $30 \mathrm{~min}$ at $37^{\circ}$ with $50 \mu \mathrm{g} / \mathrm{ml} \mathrm{fl-HDL}$, fixed with $3.75 \%$ formaldehyde and stained with different primary antibodies against organelle markers of interest and secondary antibodies labeled with Alexa-488 to be then counterstained DAPI. The following primary antibodies were used: anti-Rab5 (A) or anti-Rab7 (B), anti-Rab9 (C), anti-Syntaxin6 (D), or anti-Rab11a (F), anti-Calnexin (G) and anti-Golgi58kProtein (H).

Lysosomes and HDL were co-localized by incubation of ECs for $30 \mathrm{~min}$ at $37^{\circ}$ with $50 \mu \mathrm{g} / \mathrm{ml} \mathrm{fl-HDL}$ and during the last 10 min additionally with $1 \mu \mathrm{M}$ Lysotracker DND (E). None of the markers revealed any co-localization. white bar $=20 \mu \mathrm{m}$ 
Figure 6: HDL accumulates in large atypical organelles. A,B Endothelial cells were incubated for one hour with $1.4 \mathrm{~nm} \mathrm{Au}-\mathrm{HDL}$ at $37^{\circ} \mathrm{C}$, washed, then high pressure frozen, fixed during freeze substitution with $\mathrm{OsO} 4$ following by silver enhancement and embedding. Note the accumulation of Au-HDL in smaller and larger vesicles (A). The latter were filled with electron dense material (see magnification in B). Black bar $=500 \mathrm{~nm}$. C-G: Immunofluorescence microscopy of MVB's (C-F) and autophagosomes (G): EC Monolayers were incubated for $30 \mathrm{~min}$ at $37^{\circ}$ with $50 \mu \mathrm{g} / \mathrm{ml} 594-\mathrm{HDL}$, fixed with $3.75 \%$ formaldehyde and stained with different primary antibodies against organelle markers of interest and secondary antibodies labeled with Alexa-488 to be then counterstained DAPI. The following primary antibodies were used:, anti-CD63 (C), anti-CHMP4b (D), anti-CHMP6 (E), anti-CHMP7 $(\mathrm{F})$, and anti-LC3 $(\mathrm{G})$

Figure 7: Co-,localisation of HDL with retroendocytotic lectins within endothelial cells.In A, B and C a monolayer of ECs was first incubated for 20 min with $1 \mu \mathrm{M}$ 594-WGA and for subsequent $15 \mathrm{~min}(\mathbf{A}), 30 \mathrm{~min}(\mathbf{B})$ and $60 \mathrm{~min}$ (C) with $50 \mu \mathrm{g} / \mathrm{ml}$ 488-HDL, before cells were fixed then with $3.75 \%$ formaldehyde and counter stained with DAPI for nuclear staining. Note the initial co-localization, which disappears over time.

Figure 8: Reduced HDL uptake by pharmacological interference with the cytoskeleton or dynamin 
A monolayer of ECs was preincubated for 30 min with either DMSO alone (A) or DMSO plus $5 \mu \mathrm{M}$ Cytochalasin D (B) or $1 \mu \mathrm{M}$ Colchicine (C) or $80 \mu \mathrm{M}$ Dynasore (D) before $50 \mu \mathrm{g} / \mathrm{ml}$ 596-HDL was added and incubated for another 30 min. Cells were then fixed with $3.75 \%$ formaldehyde and counter-stained with DAPI for nuclear staining. white bar $=20 \mu \mathrm{m}$ E shows the quantification of the different conditions as described in the methods. $\mathrm{N}=5$

Figure 9: No fluid phase uptake of HDL into endothelial cells: $A$ monolayer of ECs was preincubated for 30 min with either DMSO alone $(\mathbf{A})$ or DMSO plus $10 \mu \mathrm{M}$ Amiloride (B) or or $2 \mu \mathrm{M}$ EIPA (C) before $50 \mu \mathrm{g} / \mathrm{ml} 596$ HDL was added and incubated for another $30 \mathrm{~min}$. Cells were then fixed with $3.75 \%$ formaldehyde and counter-stained with DAPI for nuclear staining. In Amiloride and EIPA treated cells, the DAPI staining was not evaluated and therefore is also not shown as both drugs show strong fluorescence in the DAPI channel.

D: Quantification of the different conditions as described in the methods $(\mathrm{N}=$ 3). For figures $\mathbf{E}, \mathbf{F}$, and $\mathbf{G}$ a monolayer of $E C s$ was incubated with $50 \mu \mathrm{g} / \mathrm{ml}$ 655-rHDL (E) together with FITC-40kDa Dextran (F) for 30 min, fixed with $3.75 \%$ formaldehyde and counter-stained with DAPI for nuclear staining. The merge does not show any co-localization (G). white bar $=20 \mu \mathrm{m}$ 


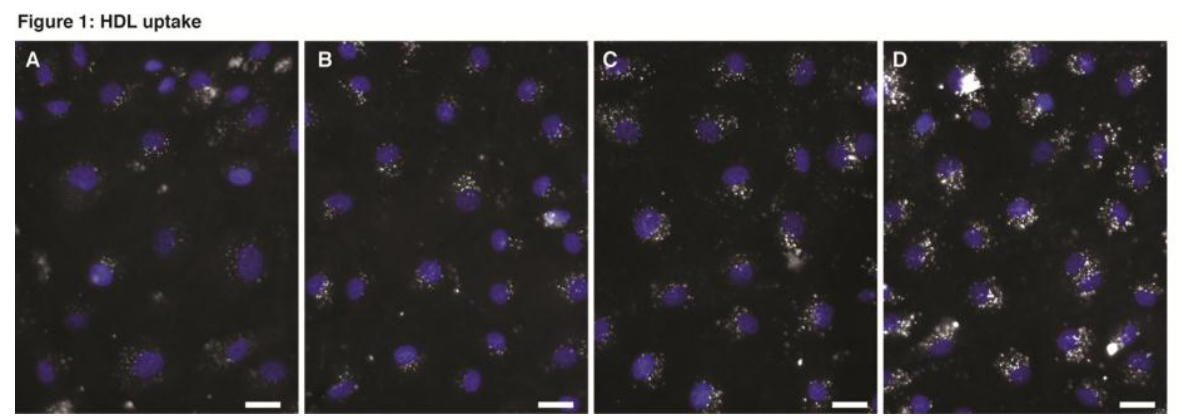


Figure 2: HDL is taken up quickly and remains inside ECs

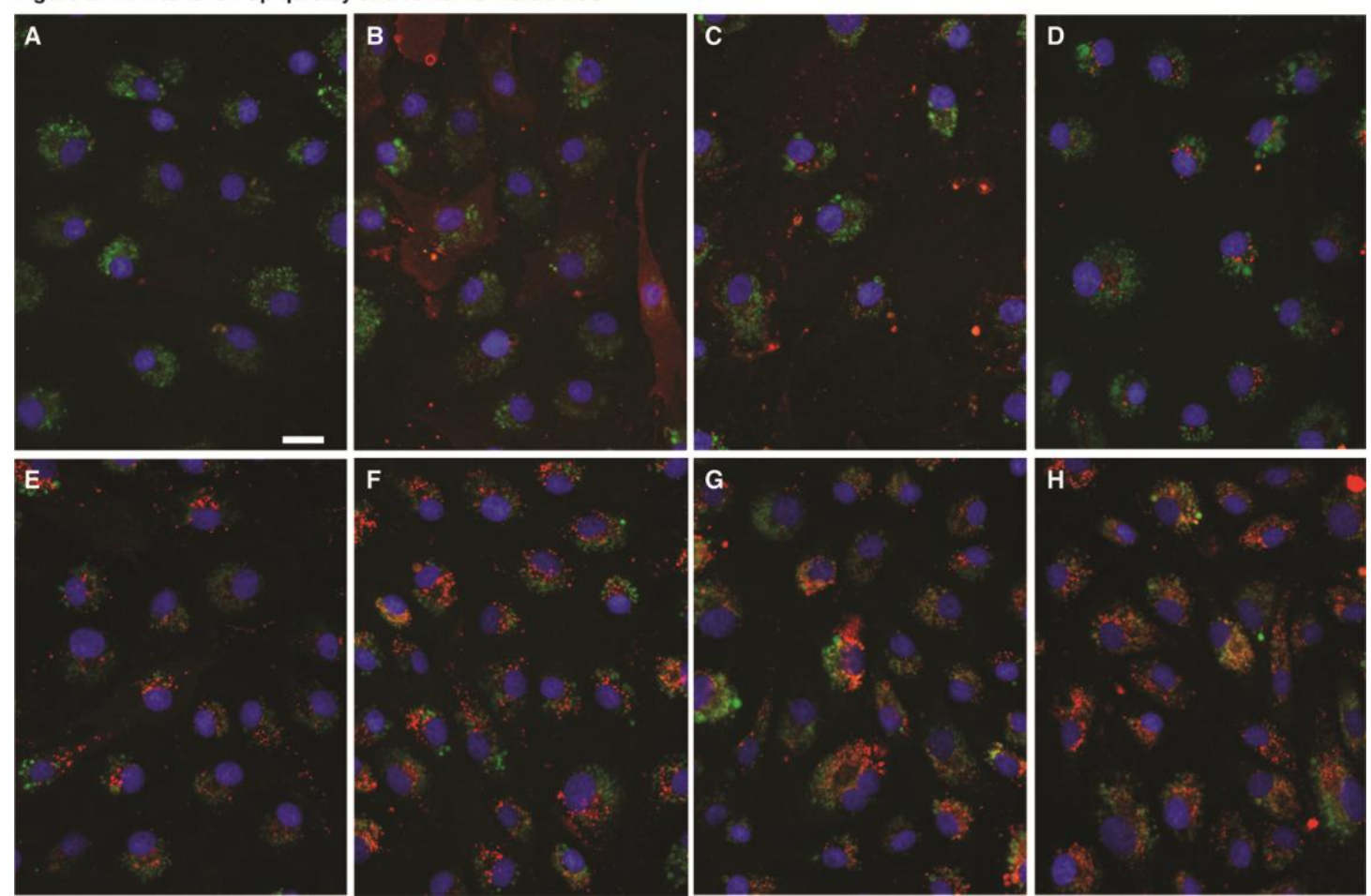


Figure 3: Lipoprotein co-localizations

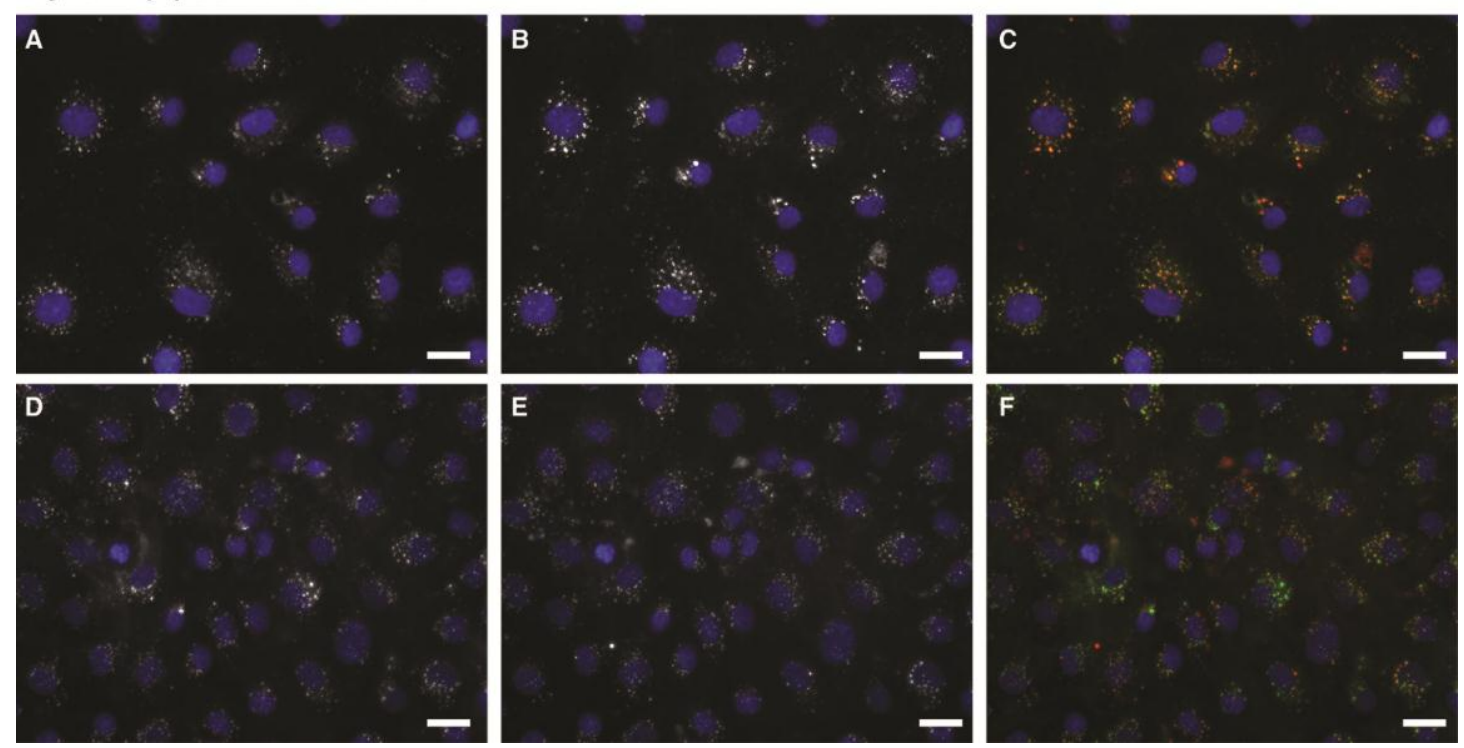




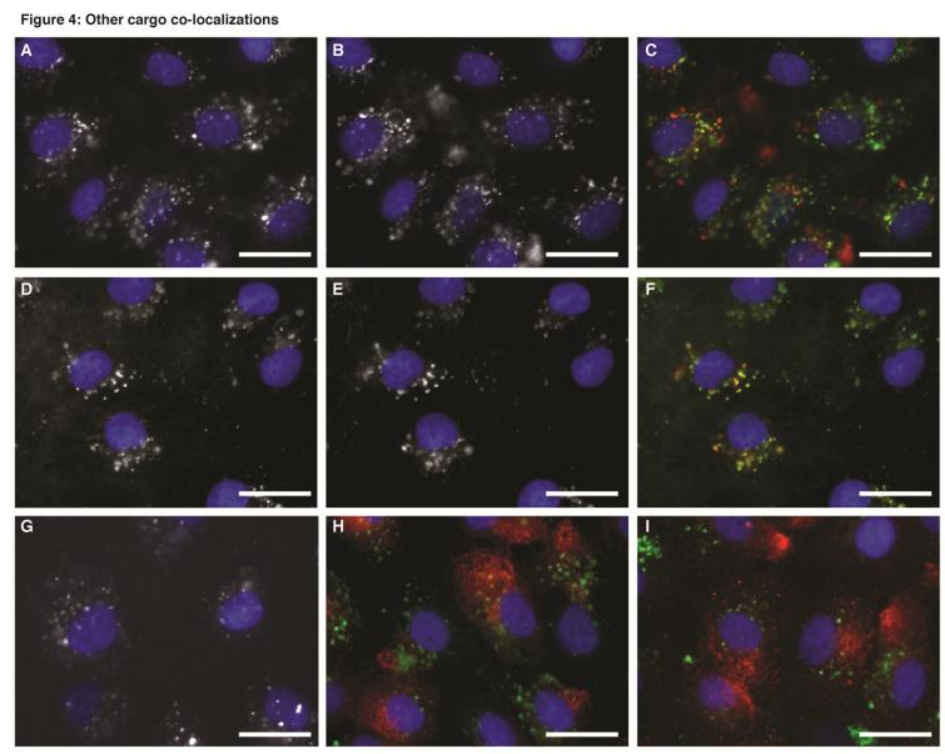


Figure 6: HDL does not colocalise with Rab5, Rab7, Rab9, Syntaxin6, Lysosomes nor Rab11b
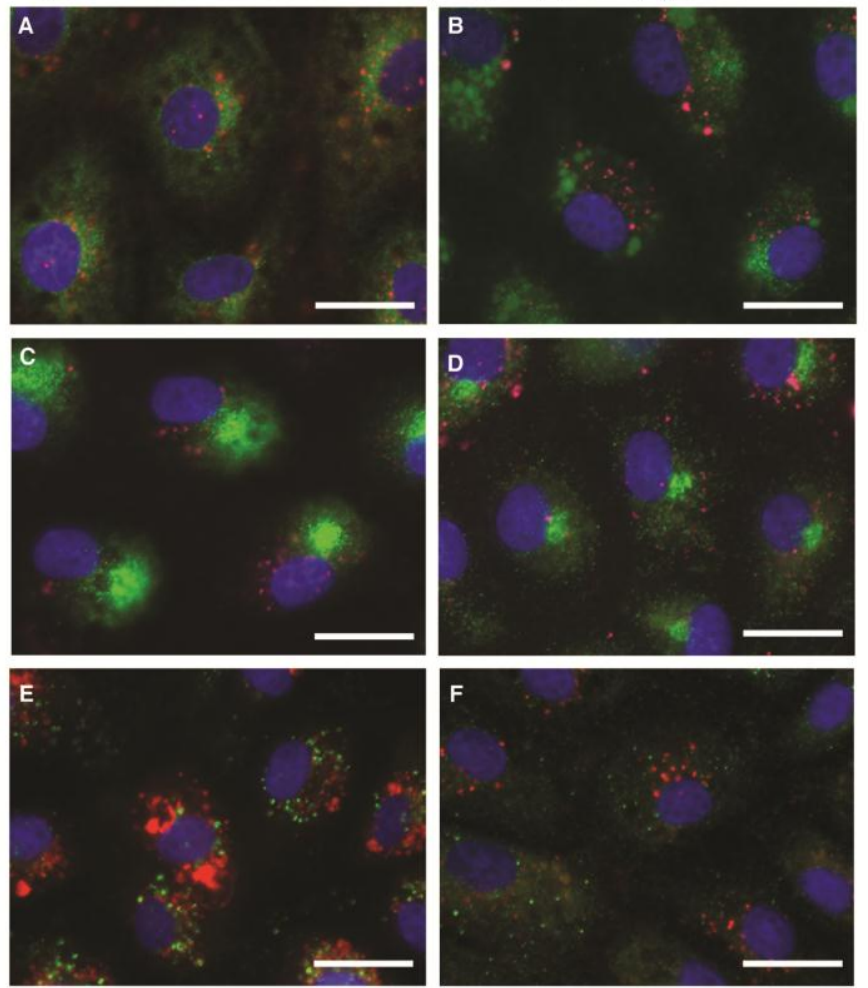

G

H
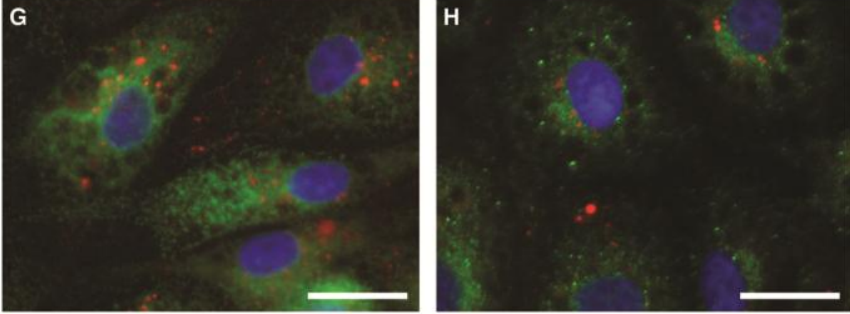


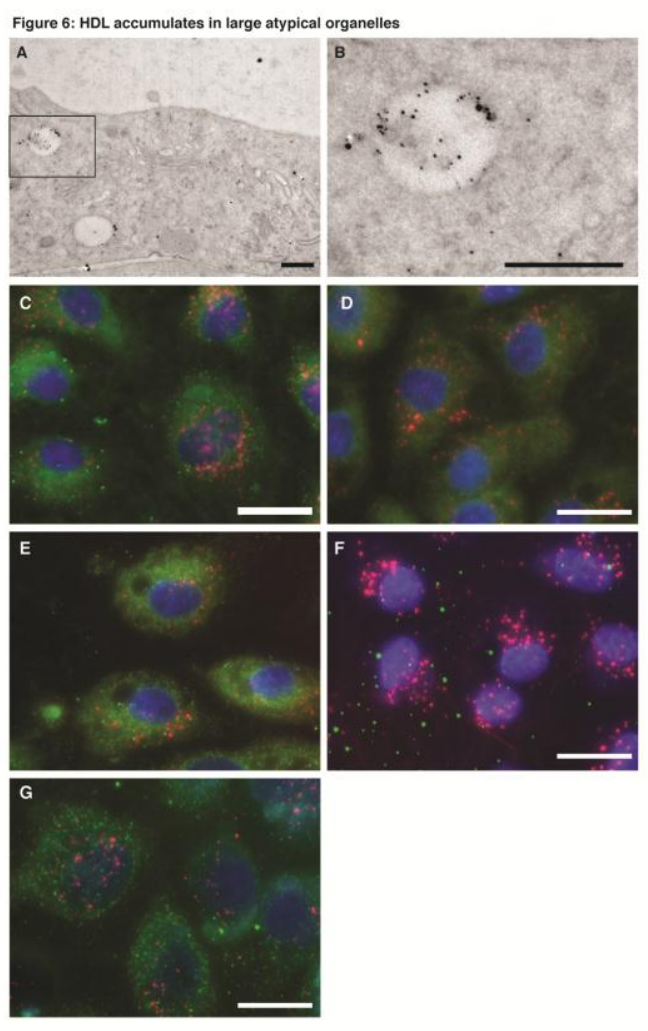




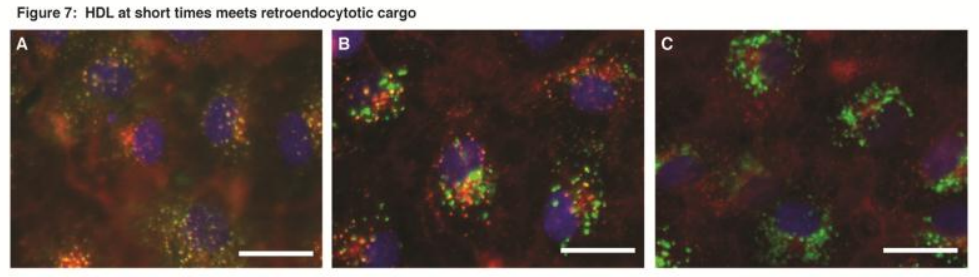


Figure 8: Pharmacological impact on HDL uptake
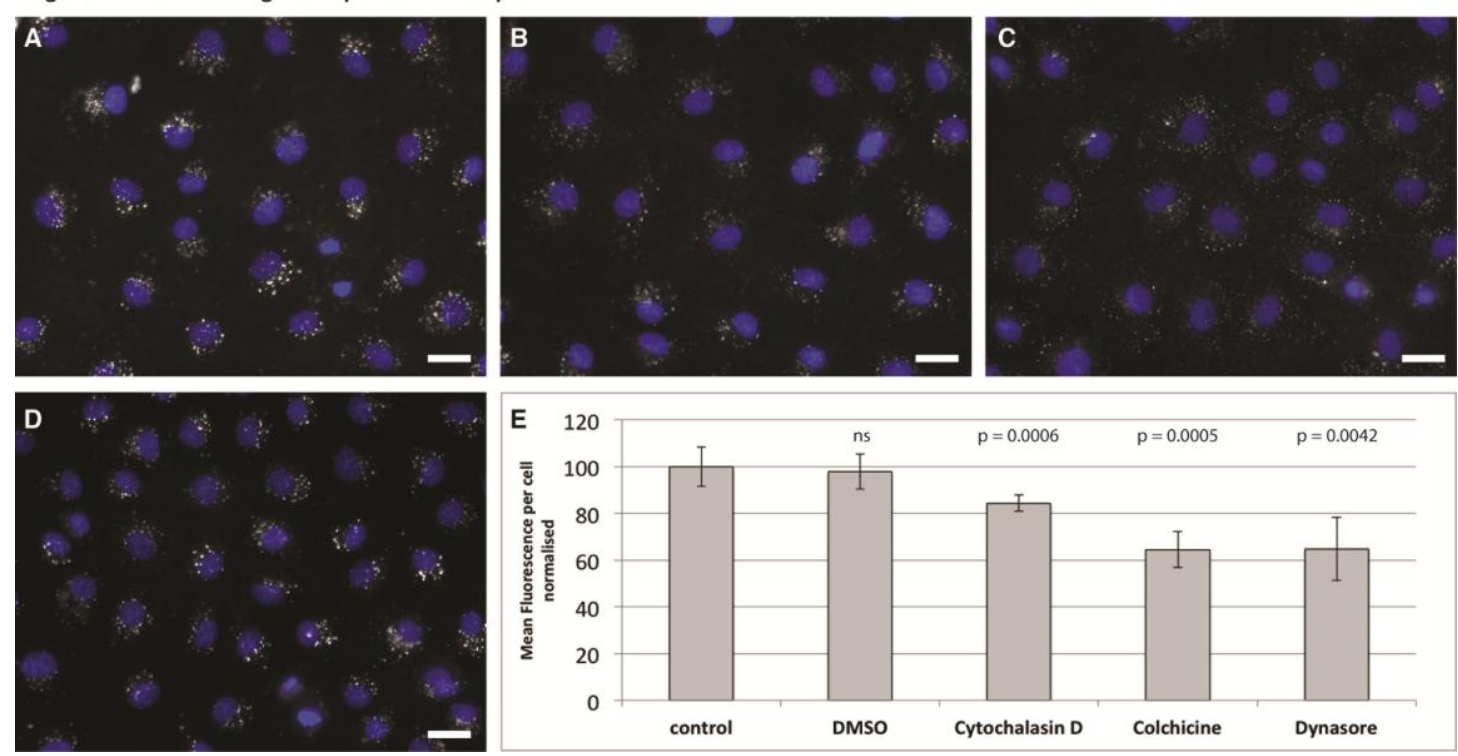


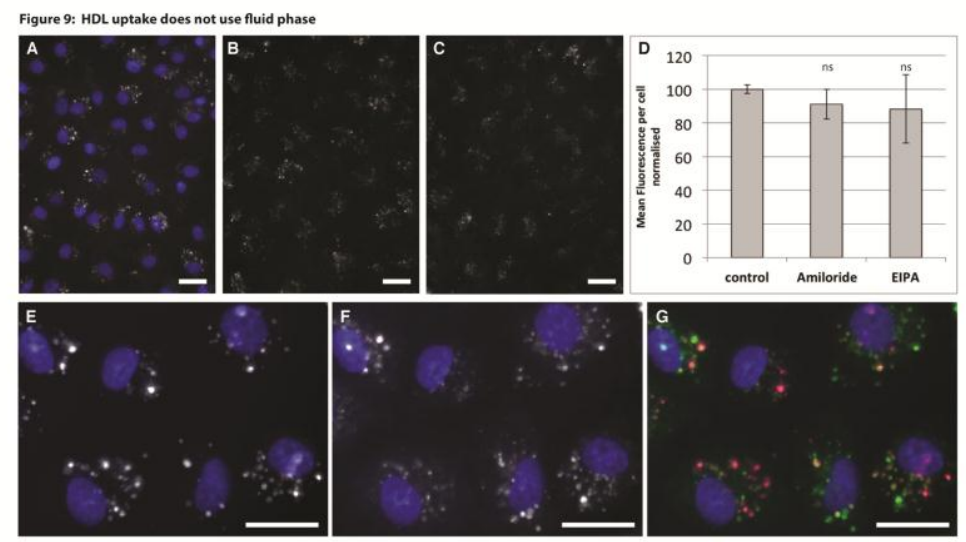

